\title{
Relation de soin médecin-patiente dans les domaines liés à la sexualité
}

Jean Martin
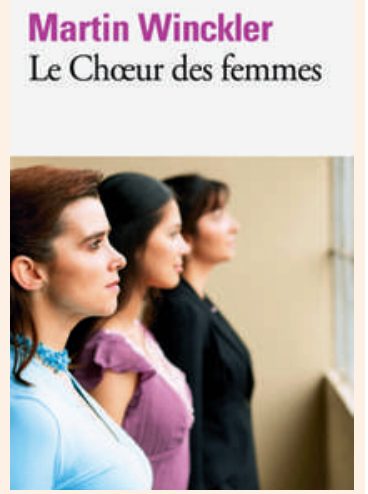

Martin Winckler Le Chœur des femmes Paris: Gallimard; 2011 682 pages. $16.90 \mathrm{CHF}$ ISBN 978-2-0704-4039-9

\section{* Médecin assistant senior, comparable à chef de clinique adjoint chez nous}

1 Martin J. Martin Winckler: la fiction qui fait approfondir un enjeu de société. Bull Méd Suisses. 2013;94(8):307-8.

jean.martin[at]saez.ch
Suis-je trop séduit par les écrits de Martin Winckler? J'ai récemment parlé dans ces colonnes de son dernier livre «En souvenir d'André» [1]; j'ai trouvé fort «La maladie de Sachs» (1998, porté au cinéma par Michel Deville en 1999), qui lui a apporté la notoriété; je l'ai vu être interviewé et j'apprécie.

De son vrai nom Marc Zaffran, Martin Winckler est un médecin français fils de médecin, né en 1955 à Alger. Il a fait ses études en France (il situe l'action de plusieurs livres dans la ville de Tourmens, en réalité Tours) puis a été durant dix ans médecin généraliste au Centre-Ouest du pays, en travaillant aussi dans un centre de consultation pour femmes. Il a beaucoup collaboré à la revue indépendante française Prescrire (dont la réputation a fait qu'elle existe maintenant en version anglaise). Ecrivain depuis l'enfance, ses romans sont largement inspirés de son expérience professionnelle.

Au cours de mon activité j'ai eu à me préoccuper de dossiers liés à la santé de la femme et de la jeune fille, surtout dans une optique de santé publique (éducation sexuelle, planning familial, interruption de grossesse et maintenant procréation médicalement assistée, abus sexuels aussi), qui ont des dimensions éthiques majeures, et ai voulu lire son ouvrage «Le Chœur des femmes». J'en propose ci-dessous des échos.

Récit de ce qu'il a appris au contact des patientes, situé dans un service lié au Centre hospitalier de Tourmens, qu'il appelle l'Unité 77 et dont le responsable est le Dr Franz Karma. La trame romancée du livre est le parcours dans cette Unité du Dr Jean (prénom anglais, = Jeanne) Atwood et des leçons qu'elle en tire. Elle est une interne* en fin de formation qui se destine à la chirurgie gynécologique et doit (au départ sans aucun plaisir) passer six mois dans un structure de soins de premier recours.

Avertissement de l'auteur: «Ce livre est un roman: les personnages, l'Unité 77, la ville de Tourmens (...) sont imaginaires. Mais presque tout le reste est vrai.» Les récits de situations cliniques, médicales et humaines, abondent.

\section{Relation soignant-soigné(e)}

Description par l'interne nouvellement arrivée de ce qu'elle observe dans la consultation du Dr Karma. Relavant d'abord sa surprise quand son chef demande à chaque patiente son accord qu'elle-même assiste à la consultation. Sur la manière: «Parfois, je le vois deviner la fin d'une phrase avant la patiente mais, au lieu de la terminer, il attend d'elle qu'elle crache la fin et, quand il voit qu'elle ne la trouve pas, il la sug- gère. Du coup ça la relance, elle se remet à raconter sa vie.» Et de montrer son impatience: «Elle m'énerve. Ils m'énervent tous les deux (...) Je n'en peux plus parce que Karma ne regarde sa montre à aucun moment. En plus, dès que la patiente lève le petit doigt, il s'interrompt, dit 〈Oui?〉 et la laisse poser une question; il n'est là que pour elle, comme s'il n'y avait pas une dizaine d'autres dans la salle d'attente.»

A propos de l'étonnement d'une femme: «Je vois bien qu'elle se demande ce qui lui arrive, si elle a bien affaire à un médecin qui a le souci de la faire avancer sans lui marcher sur les pieds et sans se formaliser qu'elle marche sur les siens.» Plus loin (c'est toujours l'interne qui commente): «Il répond gentiment (mais beaucoup trop, j'insiste) pour que ce soit honnête: aucun médecin d'aucun genre n'est aussi patient que ça avec des bonnes femmes.» Idéal, irénique, du roman...?

Le Dr Karma à propos de lutte contre la douleur: «C'est l'attitude face à la douleur qui fait la différence. Soigner ou prévenir les souffrances, c'était à l'époque bon pour les infirmières. Le rôle des médecins, c'était de faire des diagnostics (...). A la fin des années 1970, on disait encore soulager la douleur ça empêche de faire le diagnostic , donner de la morphine au cancéreux c'était dangereux parce que ça risquait de les transformer en toxicomanes.»

Quand Karma parle à sa collaboratrice de «la morgue, la boursouflure de vous-même qu'on vous a inculquée après vous avoir soigneusement humiliée» au cours des études et de la formation, il exagère, généralise indûment. L'interne réagit vivement en lui faisant remarquer qu'il n'est pas possible que les autres aient toujours tort et lui toujours raison. Mais qui nie que des postures médicales arrogantes, autoritaires, existent encore?

\section{Partenariat}

Comment savez-vous qu'elle dit la vérité? «Puisqu'elle nous l'a dit (...) Je pense que nos patientes passent leur temps à mentir mais pas toujours là où je crois; que même si elles mentent sur les détails, elles ne mentent pas quand elles expriment leurs sentiments. Depuis vingt ans, chaque fois que j'accueille une femme enceinte qui a oublié de prendre sa pilule, elle le dit avant même que j'aie posé la question parce qu'elle s'en veut. Mais celles qui ne l'ont pas oubliée sont en colère, parce qu'elles ont tout fait correctement.»

«Si vous voulez soigner, il faut vous préparer à l'idée d'être manipulé. La question est de savoir jusqu'où on se laisse manipuler, et par qui. Et on peut éviter la manipulation en passant à la coopération, au partage.» 
A propos de confidentialité et de secret, intéressantes discussions aux pages 401 et 571-572. «Un secret, c'est un symbole, pas un instrument. S'en servir, c'est s'exposer à manipuler ou à se faire manipuler (...) Le secret qu'on te confie ne t'appartient pas et il ne te confère aucun droit, aucun pouvoir, aucune autorité morale sur la personne qui te l'a livré.» Juste. hésitent mais qui se révèle un «page turner» (les pages tournent d'elles-mêmes). Engagé, décoiffant, plein de la vie des gens, de joies et de passablement de vicissitudes. Avec des indications pratiques voire techniques utiles dans les domaines de la «women's health», en particulier sur la contraception. On sent la patte de l'écrivain de romans policiers qu'il est

\section{Engagé, décoiffant, plein de la vie des gens, de joies et de passablement de vicissitudes.}

\section{Interruption de grossesse}

Winckler parle d'un contexte où bien qu'elle soit légale l'interruption volontaire de grossesse est encore sévèrement jugée par certains. Et où les médecins qui la pratiquent dans des centres publics, s'adressant surtout à des personnes peu favorisées, le font pour des motifs d'éthique sociale et de santé publique. En sachant qu'une telle interruption est toujours regrettable, est toujours un échec, d'une manière ou de l'autre, mais que cela peut être la moins mauvaise issue (à noter la description de la pratique d'une IVG, pages 153-157). Etant entendu qu'elle doit être accompagnée d'une information/éducation compréhensible et suffisante en matière de contraception. Récits aussi de drames liés aux abus sexuels et aux grossesses chez des adolescentes.

\section{Intersexualité}

A mesure que le roman déroule le parcours professionnel et humain du Dr Atwood dans l'Unité 77, apparaît sous diverses facettes la problématique intersexualité: on apprend que, plus de trente ans auparavant, un membre de sa famille, né intersexué, a été excessivement et indûment opéré par des médecins très désireux de «régulariser» rapidement un status ambigu, voulant à tort en faire une fille alors qu'il se vivait garçon; que le Dr Karma a eu connaissance de cette malpratique et que cela a déterminé son orientation professionnelle vers une médecine plus relationnelle: écouter les patients, cas échéant leurs proches, d'abord et beaucoup; avant toute action précipitée. De manière qui ne surprend pas sous la plume d'un médecin militant, on trouve dans le livre d'autres piques à l'endroit d'opérateurs qui sautent aux conclusions et à l'action..

Cette discussion a pour moi fait écho au travail récent de la Commission nationale d'éthique sur l'intersexualité [2], dont les appréciations et recommandations sont fort proches de ce qu'expose Winckler (à propos des dérives y relatives dans le passé, voir le chapitre «Jeanne», p. 493-502, et p. 650656).

Attitude à adopter face aux variations du développemen sexuel - Questions éthiques sur l'intersexualité. Berne; novembre 2012 (existe aussi en versions allemande et anglaise).

\section{Un «Chœur» substantiel et vivant}

Pavé de plus de six cent pages, longueur devant laquelle les lecteurs pas trop rapides de mon genre aussi. Ici et là un moment un rien brouillon ou pas terriblement vraisemblable.

Part de roman et part de vécu dans les écrits de notre confrère? J'ai lu plusieurs de ses livres, vu son site web et son blog (intéressants) et suis persuadé que cela ne saurait être le fruit d'un talent littéraire créatif seulement. Un bon docteur, il me semble.

Sans doute règle-t-il des comptes avec les mandarins de la corporation et un certain nombre d'idées reçues, et continue-t-il à le faire aujourd'hui. Je le note sans plaisir dans la mesure où je préfèrerais penser que la profession médicale, en France et ailleurs, a vraiment adapté ses modes de fonctionnement. Mais là aussi les faits sont têtus, s'il faut en croire par exemple les propos, très critiques de l'attitude médicale générale dans les situations de fin de vie, aujourd'hui encore, du rapport que le Prof. Didier Sicard a remis au président Hollande en décembre 2012 - rapport que Winckler commente favorablement sur son site.

Traitant de pratique médicale, de la vie des médecins et des patient(e)s, ses livres sont une lecture dynamique, agréable, fleurie ai-je envie de dire. Les praticiens expérimentés y confirmeront leurs expérience et compétences et nos confrères en formation y apprendront beaucoup. Utile, encourageant, qu'existent en français des ouvrages qui font progresser le partage adéquat d'information entre soignés et soignants et des manières partenariales d'être et de faire.

\section{Quelques formules enfin \\ (glanées au cours des pages)}

- Quand on pose des questions, on n'obtient que des réponses (ou: de l'importance d'écouter).

- Tu as le même corps que celles que tu soignes.

- Tu n'es pas responsable de ce qu'elles font, tu es responsable de ce que tu leur fais.

- Les livres de médecine ne parlent jamais des douleurs provoquées par les gestes des médecins.

- Qui soignes-tu en cet instant? Le patient ou toi?

- La loyauté d'un soignant va d'abord à ses patients, ensuite seulement à ses confrères
- Tous les patients ne sont pas aimables; mais ils n'ont pas besoin d'être aimés pour aller moins mal, ils ont besoin que tu les respectes. 